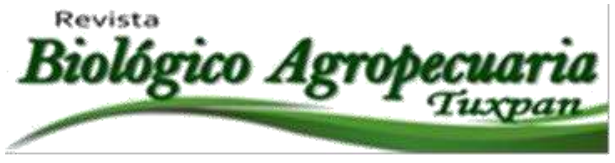

\title{
Plan estratégico regional para el desarrollo rural en el sector Agroempresarial en la zona norte del Estado de Veracruz
}

Regional strategic plan for rural development in the Agro-business sector in the northern part of the State of Veracruz

Elorza Martínez Oralia ${ }^{1}$; Rodríguez Elorza Ricardo ${ }^{2 凶}$, Álvarez Velázquez Edalid ${ }^{1}$; Flores Barrios Leonardo $^{1}$ y Cruz Luis Esteban ${ }^{1}$

${ }^{1}$ Facultad de Contaduría. Universidad Veracruzana. Zona Poza Rica-Tuxpan. ${ }^{2}$ Facultad de Ciencias Biológico Agropecuarias, Universidad Veracruzana, Carretera Tuxpan Tampico Km 7.5, Col. Universitaria, 92850 Tuxpan, Veracruz.

${ }^{\square}$ Autor para correspondencia: ricardorodriguez.e@ hotmail.com

Recibido: 18/02/2017

Aceptado: 30/06/2017

\section{RESUMEN}

Este trabajo se realizó para obtener información sobre las necesidades de las agroempresas de la Zona Norte del Estado de Veracruz. Así como también identificar las diferentes dependencias que proponen programas de apoyo monetario, con asesoramientos y capacitaciones a las agroempresas que no cuentan con una ayuda externa. Los resultados de la investigación mostraron que existen Desarrollo Rural Endógeno, Desarrollo Rural Integrado y Desarrollo Rural Territorial los cuales se enfocan al crecimiento de manera productiva y económica. También, se presentan conceptos sobre las agroempresas y sus características que la conforman. Así como también, el tamaño al que pueden crecer y el número de empleados que estas pueden emplear. Por último, se plasmó información sobre las incubadoras de negocios, con la finalidad de proporcionarles apoyos para un mejor manejo de la empresa como capacitaciones, asesorías y un manejo adecuado para el producto.

Palabras clave: Empresas, Plan de negocios, Desarrollo Rural.

\begin{abstract}
This work was carried out to obtain information on the needs of agro-enterprises in the Northern Zone of the State of Veracruz. As well as identifying the different dependencies that propose programs of monetary support, with advice and training to agribusinesses that do not have external aid. The results of the research showed that there are Endogenous Rural Development, Integrated Rural Development and Territorial Rural Development which focus on growth in a productive and economic way. Also, concepts are presented on the agroempresas and their characteristics that conform it. As well as, the size to which they can grow and the number of employees that these can employ. Finally, information
\end{abstract}


was provided on business incubators, with the purpose of providing them with support for better management of the company, such as training, advice and proper management of the product.

Keywords: Business, Business Plan, Rural Development.

\section{INTRODUCCIÓN}

El desarrollo rural es muy importante en una agroempresa debido a que estas se dedican a la transformación y comercialización de productos del campo ya sea para el sustento personal o para el público consumidor (FAO, 2016). Los Agronegocios engloban el conjunto de actividades empresariales que se llevan a cabo desde la granja hasta la mesa. Comprenden el suministro de insumos agrícolas, la producción y transformación de los productos agrícolas y su distribución a los consumidores finales. Son uno de los principales generadores de empleo e ingresos en todo el mundo (FAO, 2016).

Las políticas de la incubación de empresas se ha convertido en instrumentos populares para el desarrollo económico, nacional, y regional del país, fomentando el empleo, vinculando a la escuela empresa, creando un entorno favorable para el seguimiento de nuevas empresas y ayudando a los nuevos empresarios en la creación de nuevas empresas. La Universidad Nacional Abierta y a Distancia considera a la agroempresa como una actividad que permite aumentar y retener en las zonas rurales, el valor agregado de la producción de las economías campesinas a través de la ejecución de actividades propias de cosecha y pos cosecha en los productos procedentes de explotación agropecuaria, acuícolas, ganaderas o forestales (UNAD, 2014). Las variables más importantes de la agroempresa son : Los aspectos tecnológicos, Impacto ambiental e Aspectos culturales.

El Objetivo de este proyecto de investigación es "Identificar el desarrollo del sector agroempresarial y proyectos innovadores que permitan forjar un movimiento positivo en la economía como es la generación de empleos y permanencia de los negocios".

El estudio de mercado se llevó a cabo en la Zona Norte del Estado de Veracruz, en las localidades de Tuxpan, Alamo Temapache y Tamiahua se aplicaron encuestas con la finalidad de obtener información sobre las necesidades que presentan las agroempresas, con preguntas sobre los apoyos de las dependencias que pudieran ayudarlas y sobre los conocimientos de las diferentes instituciones y sus programas de apoyo. Asimismo, se pretende obtener información sobre el conocimiento que las agroempresas tengan sobre las incubadoras de negocios y si saben de los servicios que estas brindan así como si están dispuestos a ser apoyados por estas incubadoras de negocios.

\section{MATERIALES Y MÉTODOS}

El porcentaje de los emprendedores que están constituidas según con el número de trabajadores con el que cuenta la agroempresa. Esto quiere decir que un $33 \%$ de los entrevistados tienen una empresa del tamaño microempresa y el $67 \%$ una pequeña empresa.

La representación de las agroempresas que están constituidas legalmente y se llegó al resultado de que el total de empresas encuestadas con un $100 \%$ no están constituidas y por lo tanto el $0 \%$ con un sí. Por lo tanto, esto quiere decir que ninguna de las empresas está constituida como S.A. o S.A. de C.V. Se puede observar la mayoría de las agroempresas con un $40 \%$ que se les aplico la encuesta, están dirigidas al sector agropecuario y con el otro $40 \%$ al sector ganadero, con el 2do lugar le 
sigue la avicultura y la floricultura con un $13 \%$ y por ultimo pero no menos importante el acuícola con un 7\%.Esto quiere decir que los sectores dominantes son la ganadería y la agropecuaria. $\mathrm{El}$ porcentaje de las agroempresas que de verdad desearían tener un crecimiento en los aspectos de productividad y económicamente. Así mismo, en la gráfica de pastel con un $100 \%$ se puede apreciar que la totalidad de las empresas quiere un crecimiento y un $0 \%$ están negadas a no querer crecer. Las agroempresas suelen tener problemas de crecimiento en diferentes aspectos, por lo tanto, en esta grafica se ve que el $60 \%$ efectivamente han tenido problemas en las cuales no les permite el superarse como por ejemplo: Falta de capital para seguir produciendo, la falta de materiales que se necesiten para el trabajo y capacitación para poder dar el 2do paso y crecer. Por otra parte, el $40 \%$ no han tenido ningún problema con el crecimiento del negocio.

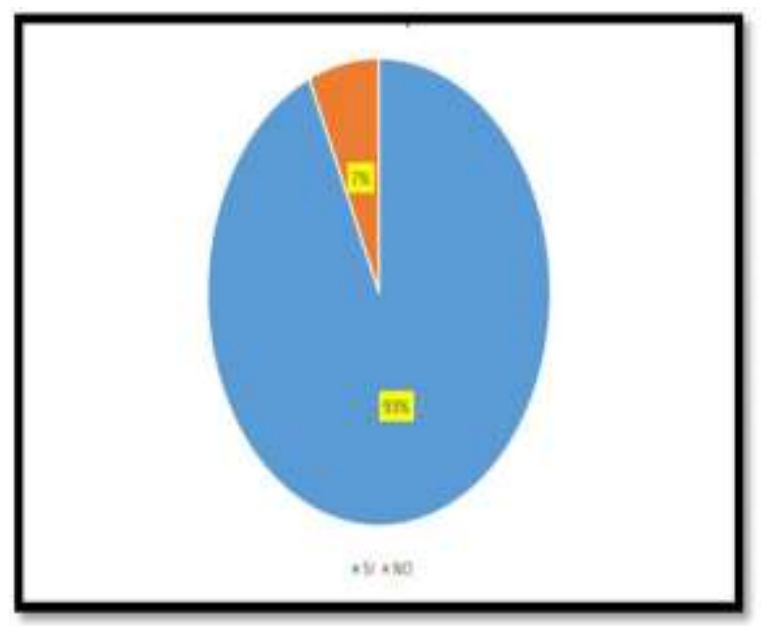

Gráfica 1. Porciento de productores que conocen los apoyos gubernamentales.

En la Gráfica 1 se muestra si las empresas cuentan con conocimiento de los programas de apoyo que imparten ciertas dependencias y con el $93 \%$ se demuestra que efectivamente se tiene conocimiento de los programas. La mayoría de estas conoce programas de SAGARPA y cuentan con el apoyo de esta dependencia. El $7 \%$ desconoce la existencia de los programas de apoyo que lanzan las dependencias de gobierno para el apoyo de las Agroempresas, esto se puede deber a la falta de información o que el emprendedor vaya iniciando un nuevo proyecto.

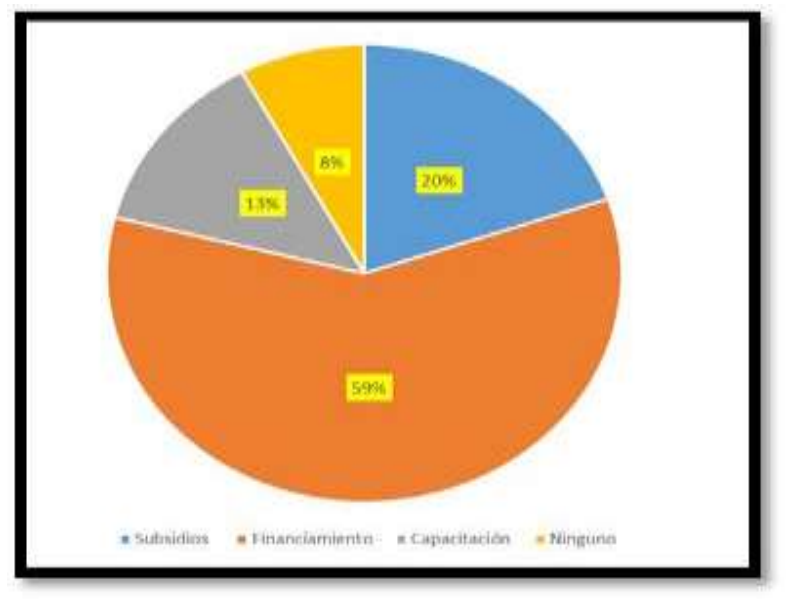

Gráfica 2. Apoyos con que cuenta su Agroempresa.

Como se observa en la Gráfica 2. El 59\% de las agroempresas encuestadas cuentan con el apoyo de financiamiento por parte de las dependencias y programas que existen, en 2do lugar con el $20 \%$ le siguen los subsidios, el 3er lugar lo lleva la capacitación con un $13 \%$ y con el $8 \%$ entran las empresas que no cuentan con dichos programas de apoyo. Esto se debe, como se había dicho anteriormente a que los emprendedores no tienen conocimiento de ayuda que puede brindar una dependencia y por lo tanto tampoco conoce los programas.

Con respecto a la Gráfica 3, encontramos que el $100 \%$ de las Agroempresas no han hecho ninguna inversión para los rubros de asesoría, capacitación y consultoría. 


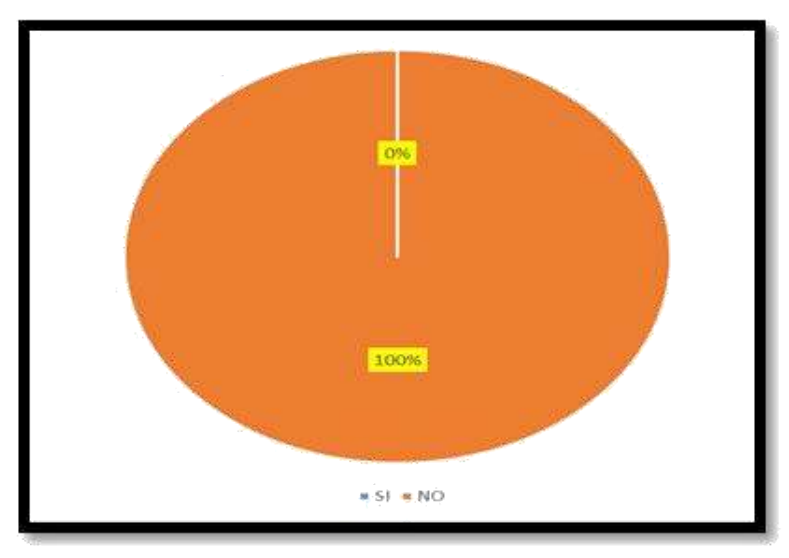

Gráfica3. Pago de asesoría, Capacitación o Consultoría.

Derivado de lo anterior el Agroempresario no tiene conocimientos de lo que es una Incubadora de Negocios, desconociendo que con esta se proporciona la mejor ayuda posible y necesaria que requiera un Agroempresa. Esto porque derivado del trabajo de campo se determinó que el $67 \%$ de las Empresas desconocen para que sirve una Incubadora de Negocios.

Con los resultados obtenidos se concluye que en el Estado de Veracruz, específicamente en la zona norte del mismo, tanto las Agroempresas nuevas como las que ya tienen un buen periodo de tiempo funcionando no todas cuentan con apoyo de las dependencias de gobierno que

existen (SAGARPA, CONAPESCA, CONAFOR, ETC). Los principales programas institucionales que coadyuvan con las Agroempresas entre otros, son: PROAGRO. Consiste en la entrega de incentivos, los cuales deben ser vinculados a mejorar la productividad agrícola. Es un programa de la Secretaría de Agricultura, Ganadería, Desarrollo Rural, Pesca y Alimentación, anteriormente era PROCAMPO pero fue reformulado el 2014. PROGRAN: Atiende a la ganadería bovina productora de carne y doble propósito en sistema de pastoreo, a la producción de leche de bovino en sistemas familiares, a la producción ovina, caprina y apícola (SAGARPA, 2015). IDETEC: Innovación para el Desarrollo Tecnológico Aplicado (IDETEC) - Maquinaria. AGROINCENTIVOS: Contribuye a incrementar la producción y productividad de las Unidades Económicas rurales agrícolas mediante incentivos para: integración de cadenas productivas (sistemas producto), desarrollo de clúster agroalimentario; inversión en capital físico, humano y tecnológico, reconversión productiva, agroinsumos, manejo postcosecha, uso eficiente de la energía y uso sustentable de los recursos naturales (SAGARPA, 2015). PIMAF - Programa de Incentivos para Productores de Maíz y Frijol. Contribuye a incrementar la producción y productividad de las unidades económicas rurales agrícolas mediante incentivos para: integración de cadenas productivas (sistemas producto), desarrollo de clúster agroalimentario; inversión en capital físico, humano y tecnológico, reconversión productiva, agro insumos, manejo postcosecha, uso eficiente de la energía y uso sustentable de los recursos naturales (SAGARPA, 2015).

CONAFOR: La Comisión Nacional Forestal, creada por decreto presidencial el 4 de abril del 2001, es un Organismo Público Descentralizado cuyo objetivo es desarrollar, favorecer e impulsar las actividades productivas, de conservación y restauración en materia forestal, así como participar en la formulación de los planes, programas, y en la aplicación de la política de desarrollo forestal sustentable.

Otro factor que se detectó entre los emprendedores encuestados fue que no están suficientemente capacitados para un manejo adecuado de su negocio, por lo tanto estos a su vez tardan años en crecer, se quedan estancados o el negocio llega a fracasar. La incubadora de negocios es sin duda una excelente manera de evitar problemas y mejorar las actividades empresariales ya que el objetivo es capacitar a los emprendedores de una manera en la cual 
puedan solucionar problemas de nivel bajo y alto, capacitación sobre la imagen de sus productos, brindar información de las dependencias con sus respectivos programas de apoyo para las Agroempresas y de manera financiera proporcionar contactos de programas que se presten a dar servicios financieros.

\section{LITERATURA CITADA}

Comps - Agruco. 2009. Desarrollo rural endógeno

http://agruco.org/compas/pdf/COMPAS\%2013. pdf. Consultado el día 22 de Agosto del 2016.

COLPOS. (2010) Desarrollo Rural.

http://www.colpos.mx/wb_pdf/Investigacion/L PI/lpi-10/PE\%20LPI\%2010.pdf. Consultado el 2 de Mayo del 2016.

CONAFOR. (2015). Comisión Nacional Forestal.

http://www.conafor.gob.mx/web/nosotros/quees-conafor/ . Consultado el dia 29 de Julio del 2016.

FAO. (2016). Desarrollo Agroempresarial. http://www.fao.org/ag/ags/desarrolloagroempresarial/vinculosagroempresariales/es/?no_cache $=1$. Consultado el 22 de Abril del 2016.

INADEM. (2016). Programa de financiamiento a emprendedores. http://www.financiamientoemprendedores.inad em.gob.mx/. Consultado el día 23 de junio de 2016

MAGRAM. 2014. Desarrollo rural integrado. http://www.magrama.gob.es/ministerio/pags/Bi blioteca/fondo/pdf/5474_8.pdf. Consultado el 22 de Agosto del 2016.

MUÑOZ. 2012. Desarrollo rural territorial. http://www.incoder.gov.co/documentos/Estrate gia\%20de\%20Desarrollo\%20Rural/Documento
s/Perfil\%20DRET.pdf. Consultado el 22 de Agosto del 2016.

PROMÉXICO. (2014). pymes, eslabón fundamental para el crecimiento en México. http://www.promexico.gob.mx/negociosinternacionales/pymes-eslabon-fundamentalpara-el-crecimiento-en-mexico.html. Consultado el día 02 de mayo del 2016.

PROMEXICO. (2016). Apoyos de promexico. http://www.promexico.gob.mx/documentos/apo yos-servicios/beneficiarios-apoyos-2014.pdf . Consultado el día 23 de Junio del 2016. 
Copyright (c) 2017 Oralia Elorza Martinez, Ricardo Rodriguez Elorza, Edalid Álvarez V elázquez,

Leonardo Flores Barrios y Luis Esteban Cruz

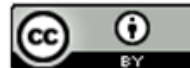

Este tex to está protegido por una licencia licencia Creative Commons 4.0.

Usted es libre para Compartir —copiar y redistribuir el material en cualquier medio o form ato-y Adaptar el documento —remezclar, transformar y crear a partir del material- para cualquier propósito,, incluso para fines comerciales, siempre que cumpla la condición de:

Atribución: Usted debe dar crédito a la obra original de manera adecuada, proporcionar un enlace a la licencia, e indicar si se han realizado cam bios. Puede hacerlo en cualquier form a razonable, pero no de form a tal que sugiera que tiene el apoyo del licenciante o 10 recibe por el uso que hace de la obra.

Resumendelicencia - Textocompletodelalicencia 